\section{Endoscopic ultrasound-guided fine needle aspira- tion diagnosis of a focal fatty mass in the pancreas}

Focal fatty masses of the pancreas, which include focal fatty infiltration, lipoma, and liposarcoma, are rare entities [1]. A well-differentiated lipogenic liposarcoma can mimic a benign lesion on radiologic examination and only cytohistology can resolve the doubt [2].

A 60-year-old woman was referred to our department because of an incidental ultrasound diagnosis of a lesion in the head of the pancreas. The patient was asymptomatic. An unenhanced computed tomography (CT) scan showed a 43-mm homogeneous, hypodense lesion at the pancreatic head $(\bullet$ Fig. 1 a). Abdominal magnetic resonance imaging (MRI) showed the lesion to be hypointense on fast imaging employing steady-state acquisition (FIESTA) with fat saturation ( $\bullet$ Fig. $\mathbf{1}$ b) and hyper- intense on T2-weighted fast-spin echo (FSE) acquisition without fat saturation (৫ Fig. 1 c). No clear contact with abdominal fat was seen.

An endoscopic ultrasound (EUS) showed a lobulated, ill-defined, heterogeneous lesion, which was slightly hypoechoic compared with the surrounding pancreatic parenchyma but with internal hyperechoic strands ( $\bullet$ Fig. $\mathbf{2 a}$ ). The appearance was compatible with both a pancreatic lipoma and focal fatty infiltration, but even a liposarcoma could not be excluded. For this reason, we decided to perform an EUSguided fine needle aspiration (FNA) with a 19-gauge needle (one passage) ( $\bullet$ Fig. $\mathbf{2 b}$ ), which revealed clearly visible fat globules on the smears ( $\bullet$ Fig. 3 a).
Cytohistologic examination showed mature adipocytes characterized by large fat droplets and eccentrically placed small nuclei without atypia. The adipocytes were interspersed with islands of normal pancreatic tissue ( $\bullet$ Fig. $\mathbf{3 b}, \mathbf{c}$ ). Immunohistochemical staining for Ki-67 showed no mitotic activity ( $\bullet$ Fig. $3 \mathrm{~d}$ ).

This focal fatty mass of the pancreatic head had some of the characteristics of a lipoma (no clear contact with abdominal fat on imaging), and some of focal fatty infiltration (no clear cytohistologic demarcation from pancreatic parenchyma) [1, 2]. This difficulty in distinguishing between these nosologic entities has been previously reported, but a clear distinction is not necessary because conservative treatment is indicated for both types of lesion. EUS-FNA, on the other hand, permits clear classification of such lesions where the differential diagnosis includes liposarcoma, which has a surgical indication. Only three other cases of EUS-FNA of a focal fatty mass of the pancreas have been reported [3-5], but considering the
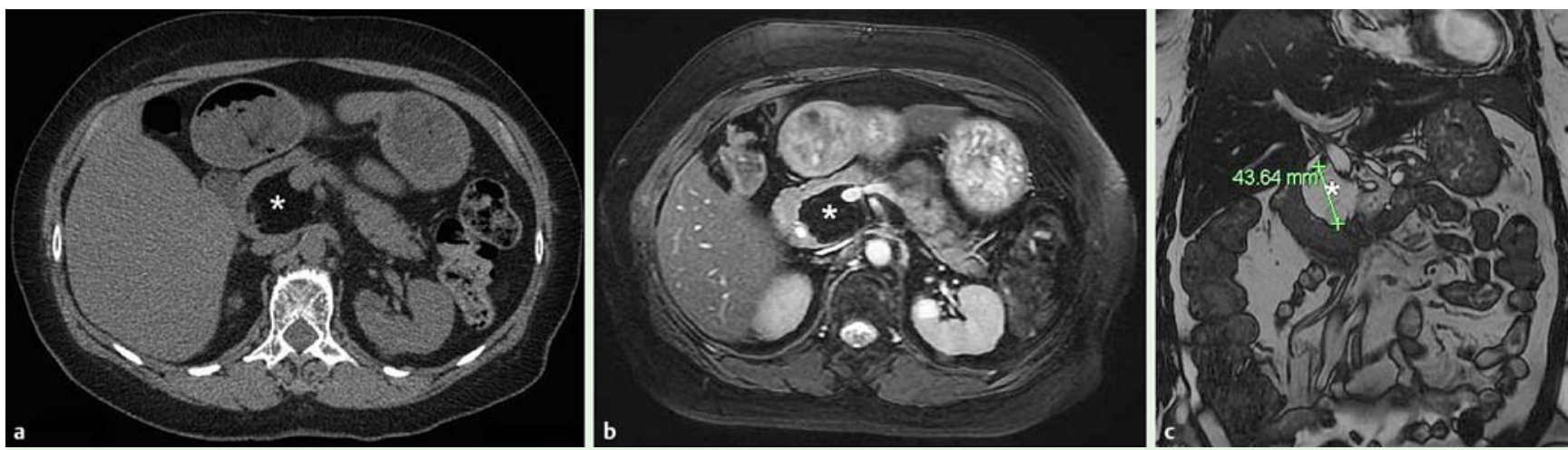

Fig. 1 Imaging of the lesion in the pancreatic head. a Computed tomography (CT) scan showing a hypodense lesion $\left({ }^{*}\right)$ in the pancreatic head. $\mathbf{b}$ Magnetic resonance imaging (MRI) scan showing the lesion is hypointense on fast imaging employing steady-state acquisition (FIESTA) with fat saturation in axial view; and $\mathbf{c}$ hyperintense on T2-weighted fast-spin echo (FSE) acquisition without fat saturation.
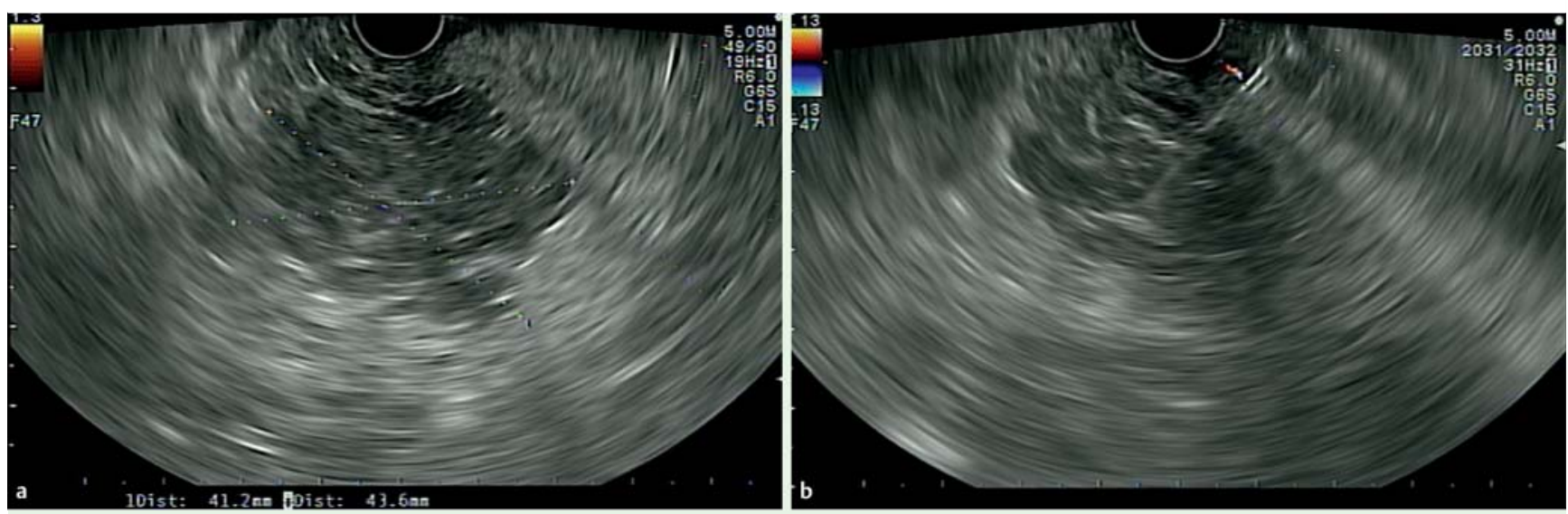

Fig. 2 Endoscopic ultrasound (EUS) images showing: a a lobulated, ill-defined, heterogeneous lesion, which is slightly hypoechoic compared with the surrounding pancreatic parenchyma but with internal hyperechoic strands; b EUS-guided fine needle aspiration (FNA) being performed with a 19-gauge needle. 


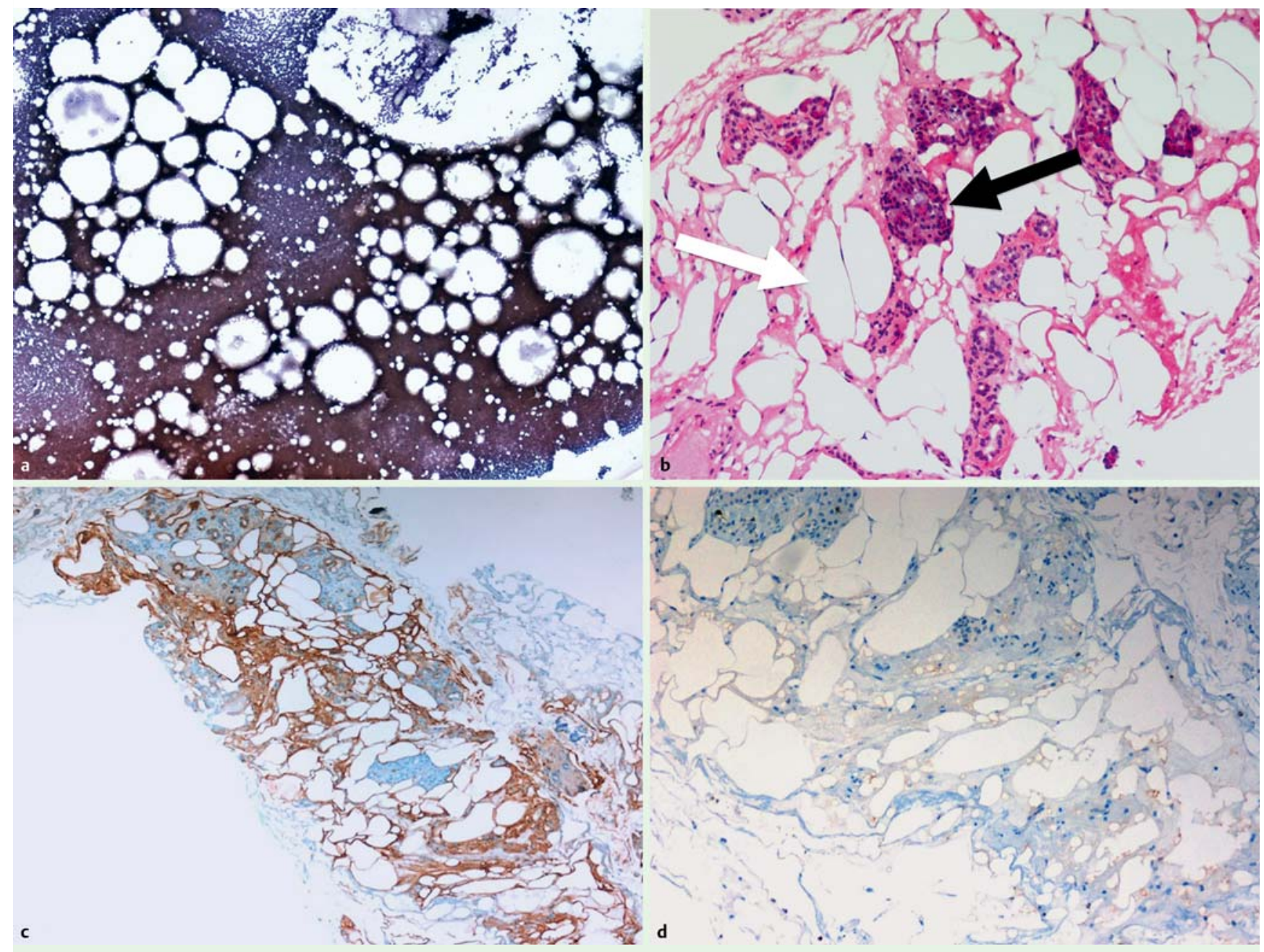

Fig. 3 Histocytological appearance showing: a on Hemacolor staining of the smear, several round empty spaces, both singly and in groups (most likely adipose tissue), with no atypical cells; $\mathbf{b}$ on a hematoxylin and eosin (H\&E)-stained section, adipose tissue (white arrow) without atypia, interspersed with islands of normal pancreatic acini (black arrow); c on an S100-stained section, adipose tissue and islands of normal pancreatic acini; $\mathbf{d}$ on Ki-67 staining, the adipose tissue is negative, indicating no mitotic activity.

low probability of complications and the potential benefit for the patient, EUS-FNA should be considered for such pancreatic lesions.

Endoscopy_UCTN_Code_CCL_1AF_2AZ_3AD

Competing interests: None

Luca Barresi ${ }^{1}$, Ilaria Tarantino ${ }^{1}$, Dario Ligresti ${ }^{1}$, Rosa Liotta ${ }^{2}$, Gabriele Curcio ${ }^{1}$, Antonino Granata ${ }^{1}$, Caruso Settimo ${ }^{3}$, Mario Traina ${ }^{1}$

${ }^{1}$ Endoscopy Service, Department of Diagnostic and Therapeutic Services, Mediterranean Institute for Transplantation and Advanced Specialized Therapies (ISMETT), Palermo, Italy

2 Pathology Service, Department of Diagnostic and Therapeutic Services, Mediterranean Institute for Transplanta- tion and Advanced Specialized Therapies (ISMETT), Palermo, Italy

Radiology Service, Department

of Diagnostic and Therapeutic Services, Mediterranean Institute for Transplantation and Advanced Specialized Therapies (ISMETT), Palermo, Italy

\section{References}

1 Secil M, Igci E, Goktay AY et al. Lipoma of the pancreas: MRI findings. Comput Med Imaging Graph 2001; 25: 507-509

2 Temizoz O, Genchellac H, Unlu E et al. Incidental pancreatic lipoma: computed tomography imaging findings with emphasis on diagnostic challenges. Can Assoc Radio J 2010; 61: 156 - 161

3 Di Matteo FM, Shimpi L, Pandolfi $M$ et al. EUS diagnosis of pancreatic lipoma. Gastrointest Endosc 2006; 64: 146-148

4 Suzuki R, Irisawa A, Hikichi T et al. Pancreatic lipoma diagnosed using EUS-FNA. A case report. JOP 2009; 10: 200-203
5 Pausawasdi N, Apisarnthanarak P, Pongpaibul $A$ et al. Pancreatic lipoma diagnosed by EUS-FNA. Gastrointest Endosc 2012; 76: $668-669$

Bibliography

DOI http://dx.doi.org/

10.1055/s-0034-1377391

Endoscopy 2014; 46: E393-E394

(c) Georg Thieme Verlag KG

Stuttgart · New York

ISSN 0013-726X

\section{Corresponding author}

\section{Luca Barresi, MD}

Gastroenterology and Endoscopy Service

ISMETT

Via Tricomi 1

Palermo 90127

Italy

Fax: +39-091-2192400

Ibarresi@ismett.edu 